Math. Model. Nat. Phenom.

Vol. 1, No. 2, 2006, pp. 45-69

\title{
Observations on the Pathophysiology and Mechanisms for Cyclic Neutropenia
}

\author{
C. Colijn ${ }^{a}$, D.C. Dale ${ }^{b}$, C. Foley ${ }^{c}$ and M.C. Mackey ${ }^{c 1}$ \\ ${ }^{a}$ Department of Engineering Mathematics, University of Bristol, Bristol, UK, BS8 1TR \\ ${ }^{b}$ Department of Medicine, University of Washington, Seattle, Washington, USA, 98195 \\ ${ }^{c}$ Department of Mathematics and Centre for Nonlinear Dynamics, McGill University \\ 3655 Promenade Sir William Osler, Montreal, QC, CANADA, H3G 1Y6
}

\begin{abstract}
We review the basic pathology of cyclical neutropenia in both humans and the grey collie, and examine the role that mathematical modeling of hematopoietic cell production has played in our understanding of the origins of this fascinating dynamical disease.
\end{abstract}

Key words: cyclic neutropenia

AMS subject classification: 92C50

\section{Introduction}

Cyclic neutropenia is a rare blood disorder usually diagnosed in children $[1,2,3]$. These children present with a history of regularly recurring fevers, mouth sores and infections. The unique feature of this disease is regularly recurring periods of severe neutropenia, usually at 21-day intervals. In most patients, the blood counts oscillate between the lower limits of normal or about $2.0 \times 10^{9} / \mathrm{L}$ at the peaks to lows of 0 to $0.2 \times 10^{9} / \mathrm{L}$ at the nadir. The count nadir usually lasts for 2 to 4 days and more severe symptoms are associated with extremely low blood neutrophil levels for longer periods. Cycling of blood monocytes from normal levels to about two or three times normal also occurs regularly with the peaks of monocytes corresponding closely to the nadir of blood neutrophils (see Figure 1). Other blood cells may also cycle, and for this reason the disease has sometimes been called "cyclic or periodic hematopoiesis" [4].

Although a rare disease, cyclic neutropenia is of broad interest for several reasons. This

\footnotetext{
${ }^{1}$ Corresponding author. E-mail: michael.mackey@mcgill.ca
} 

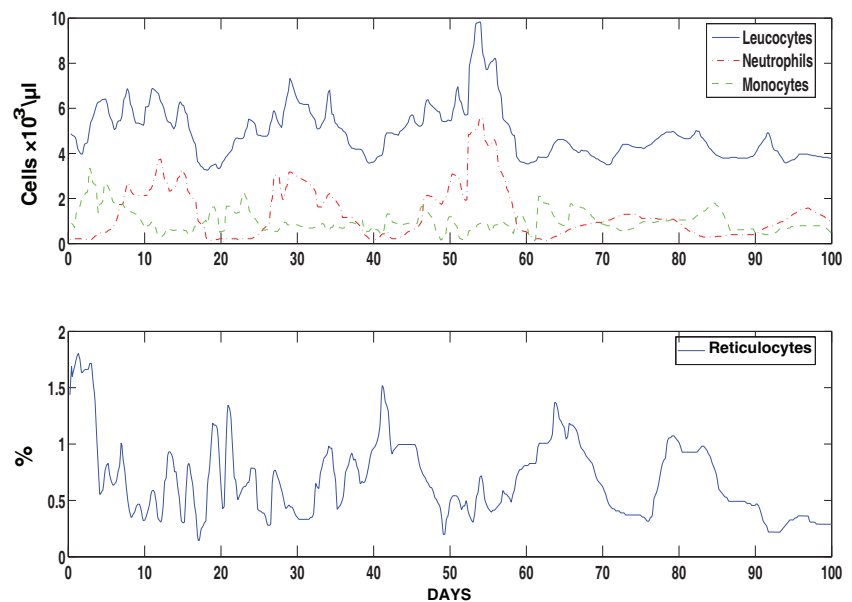

Figure 1: Blood cells count in cyclical hematopoiesis. Taken from [1].

review summarizes current understanding of the clinical, genetic and molecular aspects of the disorder and illustrates how mathematical modeling created the foundation for our current understanding of the basic mechanisms for this disorder.

\section{Clinical, Genetic and Molecular Aspects of Cyclic Neutropenia}

\subsection{Clinical features}

Cyclic neutropenia is a life long illness punctuated by episodes of severe and sometimes life threatening infections. The classic clinical features of cyclic neutropenia (mouth ulcers, periodic fever and infections) are usually observed in children before the age of 1 , but the time of onset of very regular symptoms may vary [4]. If there is careful observation or maintenance of a daily diary, the periodicity of symptoms can usually be readily plotted. In families with multiple affected members, the disease is more readily recognized than with a sporadic case. Acquired cyclic neutropenia in adulthood has been described, but such cases are very rare [1].

Deep mouth ulcers are the most characteristic clinical feature. They are very painful, often last more than a week and make eating difficult. The ulcers are quite similar to those of patients with aphthous stomatitis, a common condition affecting up to $25 \%$ of the general population [5]. However, with aphthous stomititis, the mouth ulcers occur at irregular intervals and the ulcers do not arise and heal synchronically, as occurs in cyclic neutropenia. The distinction between these entities can easily be made by performing serial blood counts; with aphthous stomatitis leukocyte and neutrophil counts are normal [5].

Patients with cyclic neutropenia develop serious infections $[1,2,3]$. During most neu- 
tropenic periods, there are symptoms of pharyngitis, sinusitis and otitis and less frequently skin infections and symptoms of a lower respiratory infection. The dread complication is abdominal pain, high fever and hypotension due to bacteremia, most frequently clostridial bacteremia [6, 7]. Myonecrosis, usually due to a mixed bacterial infection in the soft tissues requiring amputation is another severe complication.

Before the availability of the hematopoietic factors for treatment, specifically granulocyte colony stimulating factor, the standard treatments for these patients were analgesic washes for mouth ulcers, antibiotics administered promptly to patients presumed to have a bacterial infection, and surgical management for myonecrosis and perforation of colonic ulcers.

\subsection{Treatment with growth factors}

Cylical neutropenia can now be effectively treated with the hematopoetic growth factor called granulocyte colony stimulating factor or G-CSF $[8,9]$. G-CSF treatment does not eliminate cycling of blood cells, but minimizes the severity and duration of the periods of neutropenia [9].

Granulocyte colony stimulating factor is the principal regulator of neutrophil production [10]. It stimulates the formation of neutrophils from hematopoietic stem cells, accelerates the formation of neutrophils in the marrow and stimulates their delivery from the marrow into the blood. In addition, G-CSF enhances the function of mature neutrophils and promotes their survival both in vitro and in vivo [11].

The cloning of the G-CSF gene and production of this growth factor for clinical use was quickly followed by trials of G-CSF for patients with cyclic and congenital neutropenia and other diseases causing severe chronic neutropenia and susceptibility to infections [8]. A randomized controlled trial demonstrated the effectiveness of G-CSF treatment for these conditions and long-term follow-up studies have demonstrated that most patients respond well with relatively mild side effects. The adverse effects are principally bone pain and headache during the acute phase of treatment [8,12]. Patients with classic cyclic neutropenia respond readily to G-CSF at relatively low doses of about $2-3 \mu \mathrm{g} / \mathrm{kg} /$ day. This treatment shortens the duration of neutropenia. It also shortens the cycle length from roughly 21 to 14 days, an effect that is sustained over long periods of observation. With G-CSF treatment, the characteristic periodic mouth ulcers and fever are virtually eliminated and other signs of infections, and particularly severe infections, are remarkably abrogated. In some patients, osteoporosis may be a long-term complication [12].

\subsection{Genetics}

Cyclic neutropenia occurs sporadically as an autosomal dominant disorder making disease recognition and genetic counseling important in clinical care [13,4]. Through family studies and linkage analysis, the mutation causing cyclic neutropenia was localized to chromosome 19 at locus 19p 13.3, the locus for several proteases found normally in the primary granules of neutrophils [14]. Mutations in the gene for neutrophil elastase, referred to as the ELA- 
2 gene, are now recognized as causing cyclic neutropenia. In family studies, all affected individuals were found to have mutations in the ELA2 gene whereas none of the unaffected members of these families showed mutations [14].

Reimann and DeBeradinis [15] first reported that cyclic neutropenia is an inherited disease, an observation later confirmed in studies of Australian families by Morley et al. [13]. In families, the severity of symptoms and the severity of neutropenia may vary, but affected family members are generally neutropenic having neutrophil counts $<1.5 \times 10^{9} / \mathrm{L}$ on random counts whereas counts are normal, i.e. $>2.0 \times 10^{9} / \mathrm{L}$ in unaffected family members [4]. Family studies also suggest that the disease is more severe in children and is ameliorated by unknown factors as they grow older [4]. In adulthood, mouth ulcers and periodic fevers are still common, but it is often more difficult to recognize the regular cycles. Family studies and longitudinal follow-up observations [16] suggest that cyclic neutropenia is not associated with congenital abnormalities or risk of malignant transformations in the hematopoetic system or a risk of other cancers generally [4]. There is also no association with autoimmune disorders or metabolic diseases.

\subsection{Pathophysiology}

Cyclic neutropenia is attributable to oscillatory production of neutrophils and other blood cells by the bone marrow, manifested in the cycling of circulating blood cells $[2,17]$. This marrow abnormality is due to defective neutrophil formation with interruption of cell production at the promyelocyte-myelocyte stage, the stage in neutrophil development when the enzyme neutrophil elastase is normally synthesized and packaged in the neutrophil granules [18]. Several recent studies now link the finding of "maturation arrest" and the mutations in ELA2 gene. These studies all point to accelerated apoptosis of myeloid cells at the promyelocyte to myelocyte transition being the primary cellular event leading to the interruption of cell production in cyclic neutropenia. Examination of marrow samples by electron microscopy show cytoplasmic blebbing and autophagy of debris of degenerating neutrophils, features that are not observed in normal marrow samples [18]. Annexin 5 staining and fluorescence activated cell sorting (FACS analysis) show that patients have degenerating cells with exposure of phospholipids on the surface of cells which are usually maintained on the cells' internal surfaces [18]. Transfection studies utilizing several cell lines, as well as normal human CD34 cells, showed that expression of mutant ELA2 leads to the unfolded protein response with up-regulation of the master endoplasmic reticulum chaperone referred to as BiP, a molecular correlate of accelerated apoptosis.

Neutrophil elastase is synthesized and packaged in the primary granules of neutrophils [19]. It is one of the first of a family of proteases synthesized at this stage in neutrophil development, a stage marked by the expression of the surface marker CD 34. Neutrophil elastase is synthesized in the endoplasmic reticulum with a short peptide leader which appears to prevent enzymatic activity of the protein as it is synthesized. This peptide segment is then cleaved at the time of packaging of the enzyme in the primary granules of neutrophils. When released from neutrophils and their granules, neutrophil elastase has broad enzymatic 
activity. It digests elastin and a variety of other proteins including the hematopoietic growth factors [20].

In patients with cyclic neutropenia, bone marrow aspiration or biopsy at the onset of severe neutropenia characteristically shows a deficiency of cells in the late stage of neutrophil development [17]. There are fewer than normal myelocytes, metamylocytes, bands and mature neutrophils, a condition often referred to as "maturation arrest." It is significant that the break in cell development is at the pro-myelocyte to myelocyte transition, the stage at which the enzyme neutrophil elastase is normally synthesized and packaged in the primary granules. At the cellular and molecular level, mutant neutrophil elastase triggers accelerated apoptosis, either as a direct effect of the mutant protein or secondary to the unfolded protein response with induction of caspase-independent apoptosis [18, 21].

\subsection{Comparisons of cyclic neutropenia and severe congenital neu- tropenia}

Severe congenital neutropenia $(\mathrm{SCN})$ is a similar but more severe disease also attributable in most cases to mutations in the ELA2 gene. In SCN, the ELA-2 mutations are more diverse and the apoptotic process more severe, resulting in lower neutrophil counts and greater susceptibility to infections $[22,23]$. Severe congenital neutropenia is also treatable with GCSF, but it is less responsive, requiring high doses of G-CSF. In addition, SCN may evolve to acute myeloid leukemia $[16,17]$. Some patients with severe congenital neutropenia have oscillations in their blood neutrophils and these oscillations are more easily detected when they receive G-CSF therapy [24].

\subsection{Genotype-Phenotype Relationships}

Recent studies also suggest that there are specific mutations of the ELA2 gene associated with cyclic neutropenia. These mutations are now hypothesized to cause a less severe degree of accelerated apoptosis than those associated with severe congenital neutropenia. Current evidence indicates that the accelerated apoptosis of neutrophils in cyclic neutropenia is a caspase independent process [21].

Cyclic neutropenia and severe congenital neutropenia are recognized as different diseases, but the finding that mutations in the ELA-2 gene can cause both diseases prompted genotype-phenotype studies. The patterns of mutation in the two diseases shown in Figure 2. Modeling studies suggest that the mutations causing cyclic neutropenia affect the binding of neutrophil elastase to its substrates and natural inhibitors, whereas those causing congenital neutropenia may predominantly affect molecular association and folding for storage in the primary granules [21]. It is not yet clear whether specific ELA2 mutations, particularly those associated with congenital neutropenia, are associated with evolution to leukemia or if the risk of leukemia is an intrinsic risk due to more rapid turnover of progenitor cells as a response to ineffective formation of neutrophils. 


\section{ELA Mutations}

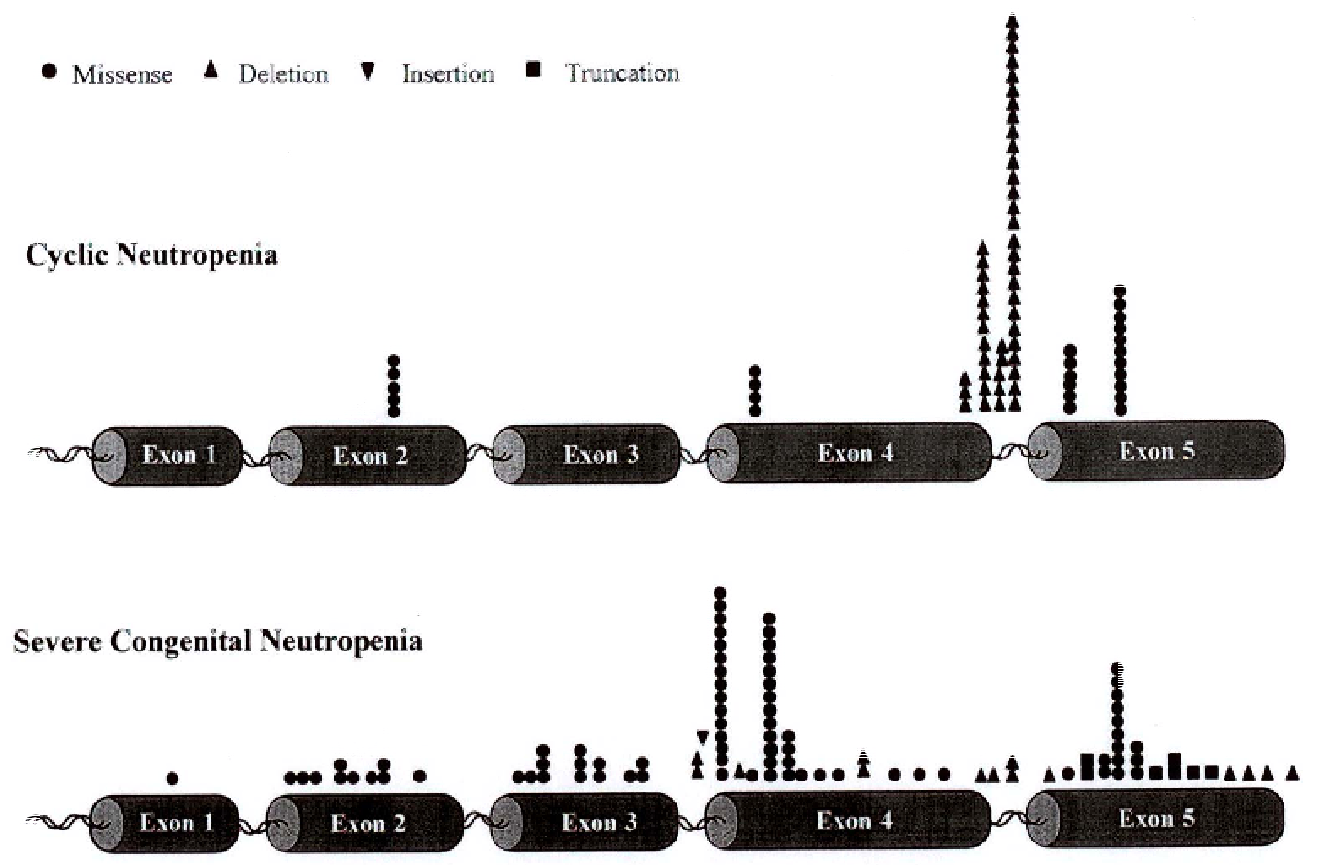

Figure 2: Mutations in the ELA-2 gene for cyclic neutropenia and congenital neutropenia. Taken from [25]. 


\subsection{Animal models}

Cyclic neutropenia or cyclic hematopoiesis also occurs in grey collie dogs [26]. The canine cycle is about two weeks [27]. The cycles are attributable to oscillations in blood cell production by the marrow and apoptosis of developing marrow cells of the neutrophil lineage [28]. In dogs the disease occurs as an autosomal recessive disorder and is attributable to mutations in the gene for AP3, a protein involved in the trafficking of neutrophil elastase into the primary granules of neutrophils [29].

The grey collie syndrome was first described in 1967 and is also referred to as the "lethal grey syndrome". Collie puppies are born with a distinctive grey coat color attributable to dilution of the melanin pigment in hair cells which also may be linked to the mutation in the gene for AP3 [29]. They also have oscillations of blood cell production with periods of neutropenia at 14-day intervals. Similar to human cyclic neutropenia, there are oscillations of other cells, most prominently blood monocytes. The marrow changes also parallel the findings in human studies [26]. There is maturation arrest of early myeloid development at the onset of neutropenia. Electron microscopic studies show cellular features of accelerated apoptosis [28]. Canine G-CSF is a very effective long-term treatment [30]. Gene therapy utilizing a lentivirus vector to induce continuous overexpression of canine G-CSF in the collie dogs is also an effective treatment; a single injection of the lentivirus G-CSF construct ameliorates neutropenia and infection for months to years [31].

\section{Mathematical models}

Mathematical modeling of the pattern of oscillation of blood neutrophils and other blood cells in patients with cyclic neutropenia suggests that cyclic neutropenia is attributable to accelerated apoptosis of myeloid progenitor cells. The original model predicted that cell loss at an early stage in neutrophil development combined with long range regulation of the blood supplies would result in oscillation of blood neutrophil levels.

Recent mathematical modeling studies suggest that cycling in blood cell production and blood cell counts may be a general phenomenon associated with ineffective production that primarily affects the early stages of cell development. In Section 3.1 we briefly review these attempts as they focus the work of this section and simultaneously motivate the extensions that we have made. In Section 3.2 we discuss models describing the coupled hematopoietic stem cells and neutrophils, and move on in Section 3.3 to models also containing the platelets and erythrocytes. In Section 3.4 we examine applications to G-CSF therapy.

\subsection{Early attempts to model cyclic neutropenia}

Given the interesting dynamical presentation of $\mathrm{CN}$ in both its clinical and laboratory manifestations, it is not surprising that there have been a number of attempts to model this disorder mathematically. 


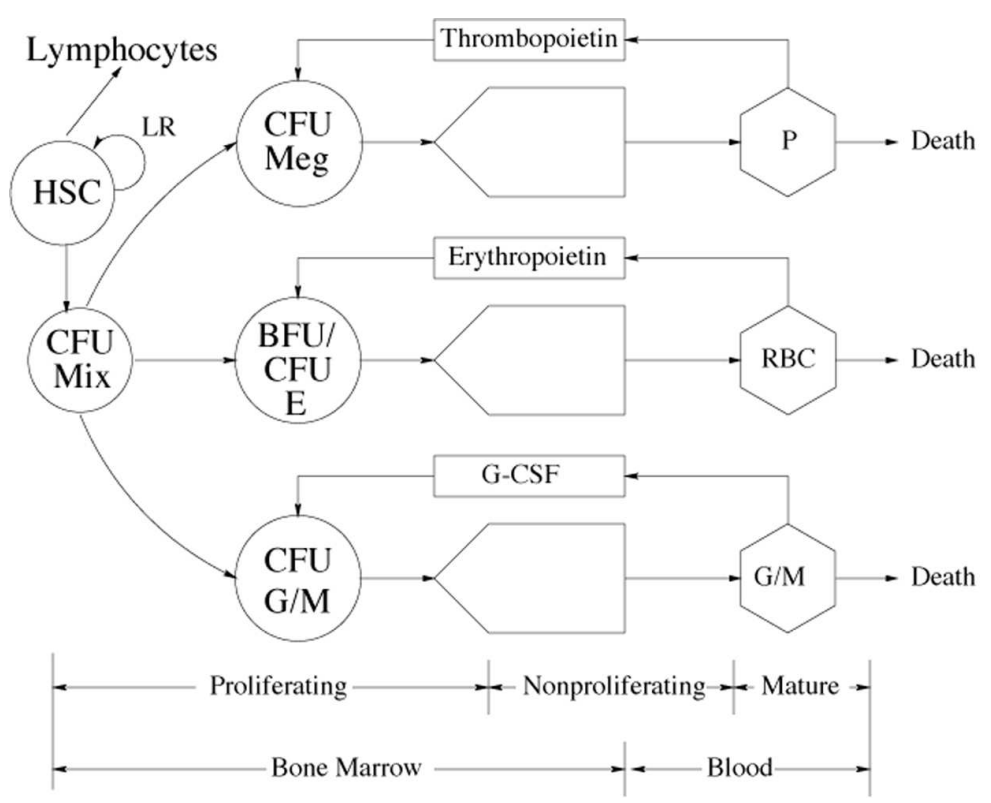

Figure 3: The architecture and control of hematopoiesis. This figure gives a schematic representation of the architecture and control of platelet $(\mathrm{P})$, red blood cell $(\mathrm{RBC})$, and monocyte (M) and granulocyte (G) (including neutrophil, basophil and eosinophil) production. Various presumptive control loops mediated by thrombopoietin (TPO), erythropoietin (EPO), and the granulocyte colony stimulating factor (G-CSF) are indicated, as well as a local regulatory (LR) loop within the totipotent hematopoietic stem cell (HSC) population. $\mathrm{CFU}$ (BFU) refers to the various colony (burst) forming units (Meg = megakaryocyte, Mix $=$ mixed, $\mathrm{E}=$ erythroid, and $\mathrm{G} / \mathrm{M}=$ granulocyte/monocyte) which are the in vitro analogs of the in vivo committed stem cells (CSC). Adapted from [35].

The mathematical models that have been put forward for the origin of CN fall into two major categories. Reference to Figure 3 will help place these in perspective. (See [32, 33, 34] for other reviews.)

The first broad group of these models identifies the origin of CN with a loss of stability in the peripheral control loop, operating as a sensor between the number of mature neutrophils and the control of the production rate of neutrophil precursors within the bone marrow (cf. Figure 3). This control has been uniformly assumed to be of a negative feedback type whereby an increase in the number of mature neutrophils leads to a decrease in the production rate of immature precursors. The other facet of this hypothesis is a significant delay due to the maturation times required between the signal to alter immature precursor production and the actual alteration of the mature population numbers. Typical examples of models of this type which have specifically considered CN are [36] - [50], all of which have postulated an alteration in the feedback on immature precursor production from the mature 
cell population numbers. These models tend to have the generic form

$$
\frac{d N}{d t}=A \mathcal{F}\left(N_{\tau_{N}}\right)-\alpha N
$$

where $N$ represents the density of circulating neutrophils, $\alpha$ is the random rate of loss of neutrophils in the circulation, $\mathcal{F}$ is the flux of cells from the stem cell compartment into the recognizable neutrophil compartment of the bone marrow, $A$ is the effective amplification through cellular proliferation within the recognizable compartment, and $\tau_{N}$ is the average length of time required to produce mature neutrophils from the entry of stem cells into the recognizable compartment. (The notation $N_{\tau_{N}} \equiv N\left(t-\tau_{N}\right)$ is used here.) $\mathcal{F}$ is typically taken to be a monotone decreasing function of $N_{\tau_{N}}$ to mimic the regulatory role of G-CSF.

The second group of models builds upon the existence of oscillations in many of the peripheral cellular elements (neutrophils, platelets, and erythroid precursors, see Figure 3) and postulates that the origin of $\mathrm{CN}$ is in the common hematopoietic stem cell (HSC) population feeding progeny into all of these differentiated cell lines. A loss of stability in the stem cell population is hypothesized to be independent of feedback from peripheral circulating cell types (see below) and would thus represent a relatively autonomous oscillation driving the three major lines of differentiated hematopoietic cells.

[51] analyzed a model for the dynamics of a stem cell population and concluded that one way the dynamic characteristics of cyclic neutropenia might emerge from such a formulation was via an abnormally large cell death rate within the proliferating compartment. This hypothesis allowed the quantitative calculation of the period of the oscillation that would ensue when stability was lost. This hypothesis has been expanded on elsewhere [52, 53] and suggested a qualitative understanding of the observed laboratory and clinical effects of G-CSF and chemotherapy that we now suspect (see below) is incomplete.

\subsection{Coupled stem cell and peripheral control loops}

A number of mathematical models coupled a stem cell compartment with peripheral control loops. In 1998, [54] developed a physiologically realistic mathematical model for granulopoiesis and examined the proposition that $\mathrm{CN}$ is due to a loss of stability in the peripheral negative feedback control of neutrophil production. Their model is a general delay differential equation (DDE) system with distributed delays. They were able to conclude that there is no consistent way in which a destabilization of the peripheral loop alone can give rise to the characteristics of $\mathrm{CN}$. It seemed more likely that the oscillations of $\mathrm{CN}$ originate from the hematopoietic stem cell population as was originally proposed in earlier work by [51, 52]. Then, [55] modified the general model of [54] by writing out explicitly the form of the control mechanism. In particular, they explicitly estimated the amplification rate of cells entering the recognizable neutrophil precursor pool (including apoptosis) as well as the form of the negative feedback loop mediated by endogenous G-CSF.

They used an oscillatory stem cell input to the neutrophil regulatory system and argued that the origin of the oscillatory behavior in cyclic neutropenia is in the stem cell population, 
consistent with experimental data and evidence. This would also explain the fact that in $\mathrm{CN}$, other cell lineages oscillate with the same period as the neutrophils [34, 55].

Those two models naturally lead to the model presented in [56]. The aim of this work was to better understand the origin of cyclic neutropenia in the HSC compartment. In particular, they were interested in the mechanisms by which the oscillatory input suggested in [55] could account for the oscillations seen in cyclic neutropenia. They built a two dimensional delay differential equations (DDE) system in which they replace the previous oscillating input by a stem cell compartment regulated by a negative feedback loop. Hence, the new model has negative feedback loops in both the peripheral loop and the stem cell loop. Using a combination of mathematical analysis and computational tools, they showed that an increased apoptosis rate in the neutrophil precursors leads to oscillations in the HSC compartment.

\subsubsection{Description of the model}

In Figure 4, the two compartments of the model are illustrated: the hematopoietic stem cell (HSC) compartment (denoted $S$ ) and the neutrophil compartment (denoted $N$ ). The HSCs are assumed to be self-renewing and pluripotential, i.e. they can differentiate into any blood cell lineage. Hence, cells in the resting $\left(G_{0}\right)$ phase can either enter the proliferative phase at rate $K(S)$ or differentiate into neutrophils $(N)$ at rate $F(N)$. As the neutrophil precursors differentiate, their numbers are amplified by a factor $A$, which accounts for both successive divisions and cell loss due to apoptosis. It is also assumed that apoptosis occurs during the proliferative phase at rate $\gamma_{s}$ and that mature neutrophils die at rate $\alpha$. As can be seen in Figure 4 , the system is controlled by two negative feedback loops. The first one regulates the rate $K(S)$ of reentry of HSCs to the proliferative cycle, and it operates with a delay $\tau_{s}$ that accounts for the time required to produce two daughter cells from one mother cell. The second loop regulates the rate $F(N)$ of HSC differentiation into mature neutrophils. It operates with a delay $\tau_{N}$ that account for the transit time through the neutrophil precursor compartment.

Mathematically, this model translates into a two-dimensional delay differential equation (DDEs) form. The equations for the two variables $N$ and $S$ can be written from Figure 4. For the compartment $N$, the loss is the efflux to death $\alpha N$. The production of mature neutrophils is equal to the influx $F(N) S$ from the HSC compartment times the amplification $A$. However, one needs to take into account the transit time $\tau_{N}$, so that the total production of mature neutrophils is $A F\left(N\left(t-\tau_{N}\right)\right) S\left(t-\tau_{N}\right)$, or equivalently $A F\left(N_{\tau_{N}}\right) S_{\tau_{N}}$ (recall that $\left.N_{\tau_{N}}=N\left(t-\tau_{N}\right)\right)$. This leads to the total rate of change of $N$ :

$$
\frac{d N}{d t}=-\alpha N+A F\left(N_{\tau_{N}}\right) S_{\tau_{N}}
$$

For the second variable, the loss from the compartment $S$ is the flux reentering the proliferative phase, $K(S) S$, plus the efflux going into differentiation, $F(N) S$. The production of $S$ is equal to the flux of cells reentering and surviving the proliferative phase, given by 


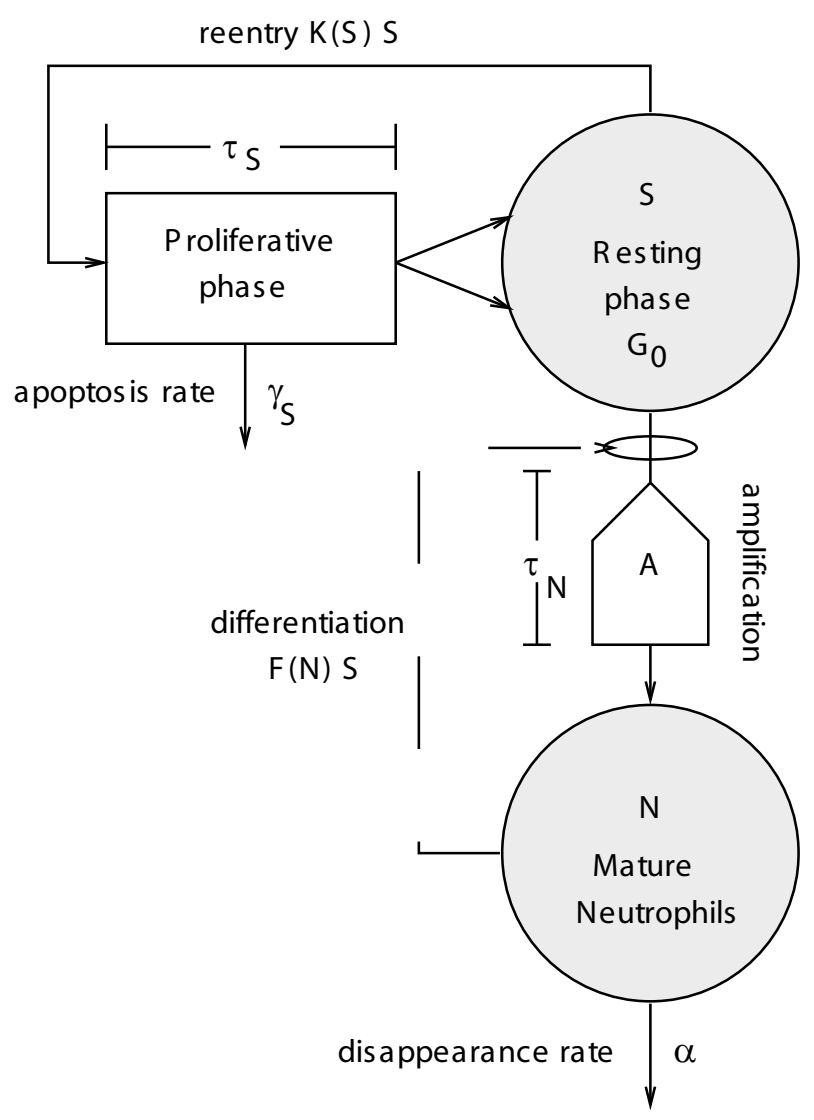

Figure 4: Schematic representation of the mathematical model. Two feedback loops control the entire process through the proliferation rate $K(S)$ and the differentiation rate $F(N)$. Taken from [56]. 
$K\left(S_{\tau_{S}}\right) S_{\tau_{S}} e^{-\gamma_{S} \tau_{S}}$, times the cell division factor 2 . The dynamics of $S$ is then described by

$$
\frac{d S}{d t}=-F(N) S-K(S) S+2 K\left(S_{\tau_{S}}\right) S_{\tau_{S}} e^{-\gamma_{S} \tau_{S}}
$$

The feedback functions $F(N)$ and $K(S)$ are monotone decreasing Hill functions:

$$
F(N)=f_{0} \frac{\theta_{1}^{n}}{\theta_{1}^{n}+N^{n}}
$$

and

$$
K(S)=k_{0} \frac{\theta_{2}^{s}}{\theta_{2}^{s}+S^{s}} .
$$

$F(N)$ controls the number of neutrophils $(N)$ while $K(S)$ regulates the level of HSCs $(S)$.

The model is sufficiently simple that the authors were able to perform mathematical analysis and a complete bifurcation analysis. In [56], they performed a complete mathematical analysis of the model that highlighted its dynamical features. Using bifurcation analysis, they showed that the origin of cyclic neutropenia is probably due an increased apoptosis rate in the recognizable and committed neutrophil precursors, leading to a destabilization of the hematopoietic stem cell compartment through a Hopf bifurcation. As a result, the HSCs start oscillating, which provides the kind of oscillatory input to the neutrophil compartment postulated by [55].

In this model, the effects of the cytokine G-CSF have been included implicitly through the feedback function $F(N)$ and are based on a pseudo-equilibrium assumption on the kinetics of G-CSF clearance, which is a simplification.

\subsection{Comprehensive models of the hematopoietic system}

As mentioned, both platelet and reticulocyte numbers can show oscillations in CN. This, together with the promise of models such as developed in [56] in explaining the qualitative features of $\mathrm{CN}$, motivated the development of models that include not only the hematopoietic stem cells and the neutrophils, but also the platelets and erythrocytes. The inclusion of the platelets and erythrocytes permits the comparison of the model simulations to observed data, not only because the platelet counts are represented in the model, but because having additional demands on hematopoietic production makes the neutrophil differentiation levels more realistic.

[57] present a comprehensive model that contains these four cell types. The stem cell and neutrophil dynamics are based on the model in [56], and the erythrocyte and platelet compartments are simplified models based on [58] and [59] respectively. The circulating cells are coupled to each other via their common origins in the stem cells, together with the regulatory feedbacks, which determine how much differentiation there is from the stem cells into each lineage at any time. It is assumed that the numbers of circulating cells in each lineage affect the amount of differentiation into that lineage, as in Eq. 3.2 above, and again, it takes several days for newly differentiated precursor cells to reach maturity. This results 
in delays in each of the feedback functions. Thus, the model consists of a set of four coupled delay differential questions. The derivation is similar to Eqs. 3.2 and 3.3, with additional delayed feedback loops regulating the erythrocyte and platelet numbers:

$$
\begin{aligned}
& \frac{d Q}{d t}=-\beta(Q) Q-\left(\kappa_{N}+\kappa_{R}+\kappa_{P}\right) Q+2 e^{-\gamma_{S} \tau_{S}} \beta\left(Q_{\tau_{S}}\right) Q_{\tau_{S}} \\
& \frac{d N}{d t}=-\gamma_{N} N+A_{N} \kappa_{N}\left(N_{\tau_{N}}\right) Q_{\tau_{N}} \\
& \frac{d R}{d t}=-\gamma_{R} R+A_{R}\left\{\kappa_{R}\left(R_{\tau_{R M}}\right) Q_{\tau_{R M}}-e^{-\gamma_{R} \tau_{R S}} \kappa_{R}\left(R_{\tau_{R M}+\tau_{R S}}\right) Q_{\tau_{R M}+\tau_{R S}}\right\} \\
& \frac{d P}{d t}=-\gamma_{P} P+A_{P}\left\{\kappa_{P}\left(P_{\tau_{P M}}\right) Q_{\tau_{P M}}-e^{-\gamma_{P} \tau_{P S}} \kappa_{P}\left(P_{\tau_{P M}+\tau_{P S}}\right) Q_{\tau_{P M}+\tau_{P S}}\right\} .
\end{aligned}
$$

Analogous to Eq. 3.4 and 3.5 we have

$$
\begin{aligned}
\beta(Q) & =k_{0} \frac{\theta_{2}^{s}}{\theta_{2}^{s}+Q^{s}} \\
\kappa_{N}(N) & =f_{0} \frac{\theta_{1}^{n}}{\theta_{1}^{n}+N^{n}} \\
\kappa_{P}(P) & =\frac{\bar{\kappa}_{p}}{1+K_{p} P^{r}} \\
\kappa_{R}(R) & =\frac{\bar{\kappa}_{r}}{1+K_{r} R^{m_{e}}}
\end{aligned}
$$

where the first two functions are the same as in [56]. In the previous equations, $Q(t), N(t)$, $R(t)$ and $P(t)$ denote the circulating number of stem cells, neutrophils, erythrocytes and platelets respectively. A detailed development of this model is given in [57].

In [60] this model was applied to cyclic neutropenia. The goal was to test whether the hypothesis of [54] and [55] still holds in this new system. The authors used a simulated annealing approach to automated fitting of model simulations to clinical data for both human and gray collie serial neutrophil and platelet counts. The model predicted that realistic CN neutrophil and platelet oscillations can result from an increase in apoptosis among neutrophil precursors. While this was the central change necessary to mimic data, it was also necessary to decrease the rate of differentiation into the neutrophil line and the maximal rate of reentry of the stem cells into the proliferative phase. The authors took the same approach to modeling CN under treatment with G-CSF and found that under G-CSF treatment the rate of apoptosis among neutrophil precursors decreases back to normal levels and the amount of differentiation into the neutrophil line rises. Both are consistent with the effects of G$\mathrm{CSF}$ as described in Section 2.2. In addition, the death rate among the proliferating stem cells must also increase in order for the model to mimic observed oscillations in treated CN. Alterations in stem cell apoptosis may be related to the connections between neutropenia and leukemia (see Section 2.5). Furthermore, changes in the stem cell death rate were previously hypothesized as explanations for the features of treated and untreated CN (see Section 3.1). 
The model given in [60] has also been analyzed for its stability and bifurcation structure [61]. It predicted that while the parameter changes resulting from G-CSF treatment will decrease the period of oscillation, they will not always abolish oscillations. This is consistent with observation. Furthermore, the model has several distinct locally stable (and hence possible) periodic solutions, as well as a locally stable equilibrium (see Section 3.4 for a discussion of multistability in the model of [56]). This presents the possibility that the same individual may experience distinct long-term outcomes as a result of different initial conditions, or different temporary interventions.

The model predicted that changes in the platelet compartment can have long-term effects on the nature of the oscillations. Simulations show that temporarily increasing $A_{P}$, the amplification parameter in the platelet compartment, will often induce the simulations to jump from an oscillating solution to the coexisting stable solution. Oscillations are thereby abolished. While there are limitations to the clinical applicability of these results because of the difficulties in administering a drug such as thrombopoietin, the ability of the platelet dynamics to affect the long-term behavior of the whole hematopoietic system is theoretically intriguing.

\subsection{Using mathematical models to explore patterned G-CSF ther- apy}

While G-CSF alleviates the most severe features of CN, it does not in general abolish oscillations in the neutrophils. Furthermore, it is relatively expensive and can have significant side effects. These facts, and the existence of several possible long-term outcomes (locally stable behaviors) for some individuals, motivate modeling the effects of treatment schedules in an effort to better understand how G-CSF may be optimally used.

\subsubsection{Optmizing G-CSF treatment with a reduced model}

We first show how the model presented in Section 3.2 has been used to study the optimization of G-CSF treatment for grey collies. [62] analyzed this model with respect to G-CSF treatment and studied the efficacy of four different G-CSF treatment protocols for CN; computer simulations predicted that clinically desirable results could be achieved using less G-CSF than would be needed with the standard daily treatment.

As has been mentioned in Section 3.2, there is no parameter in this reduced model that explicitly represents G-CSF. However, [56] showed that by using physiologically relevant parameter values, their model can replicate the characteristics of $\mathrm{CN}$ and the effects of G$\mathrm{CSF}$ administration. Mimicking $\mathrm{CN}$ can be achieved by increasing the rate apoptosis for the neutrophil precursors, i.e. decreasing the amplification parameter $A$ (which accounts for cell death). To simulate G-CSF in CN the authors modified five of the eleven parameters of the model. First, under G-CSF, apopotosis in both the HSC (decrease $\gamma_{s}$ ) and the neutrophil precursors (increase the amplification $A$ ) was decreased. Second, the durations of both the proliferative phase (decrease $\tau_{s}$ ) and the differentiating phase (decrease $\tau_{N}$ ) were decreased. 


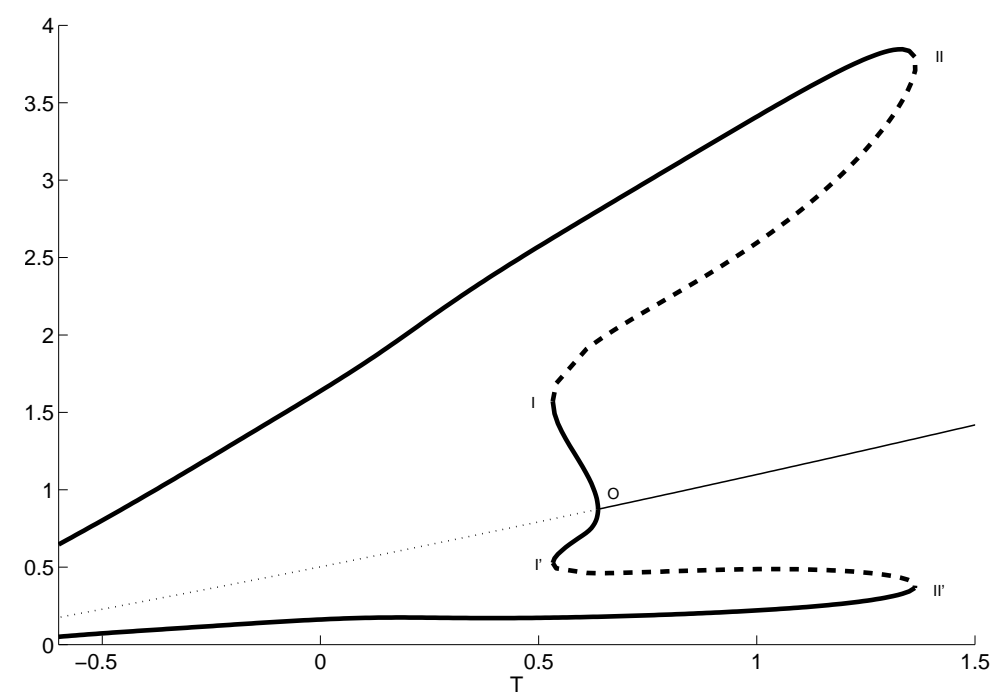

Figure 5: Bifurcation diagram for $N$ with respect to $T$. The envelope of the solution (thick line) as well as the fixed point (thin line) are shown. A Hopf bifurcation occurs at point $O$, where the stability of the fixed point is changed and a small stable periodic orbit is created. This limit cycle exists between $T=0.53$ and $T=0.63$, before disappearing through a reverse saddle-node bifurcation at point $I$, together with an unstable limit cycle. This unstable limit cycle exists between $T=0.53$ (points $I, I^{\prime}$ ) and $T=1.36$ (points II,II'), where it was created through a saddle-node bifurcation. Notice that there is a bistability for $T$ between 0.63 and 1.36. From [62].

Finally, the differentiation rate $F(N)$ was increased by increasing the parameter $\theta_{1}$, which is proportional to G-CSF production. Thus, in this model there were two sets of parameter values of interest: for a dog suffering from $\mathrm{CN}$ (1 parameter modified) and for a $\mathrm{CN}$ dog under daily G-CSF treatment ( 5 parameters modified). For more details about the estimation of parameters, see [56] and [62].

To obtain more insight into the dynamical features of the model during G-CSf treatment, the authors performed a bifurcation analysis. However, performing a bifurcation analysis with respect to five parameters is hard to interpret and carry out. Hence, the authors assumed that the five parameters were varying linearly between the untreated CN state and the G-CSF treated CN values. They expressed the five relevant parameters $\left(A, \theta_{1}, \tau_{s}, \tau_{N}\right.$ and $\gamma_{s}$ ) in terms of a new parameter $T$ such that $T=0$ corresponds to untreated CN and $T=1$ to G-CSF treated $\mathrm{CN}$ respectively. Increasing $T$ was therefore associated with increasing G-CSF concentration. In Figure 5, the bifurcation diagram for $N$ with respect to this new parameter $T$ is presented.

Much information about the dynamical features of the model can be gleaned from Figure 5. First, at $T=0$, the steady state is unstable (dotted line). A large amplitude stable periodic orbit (thick solid line) exists and accounts for the oscillations seen in CN. As $T$ 

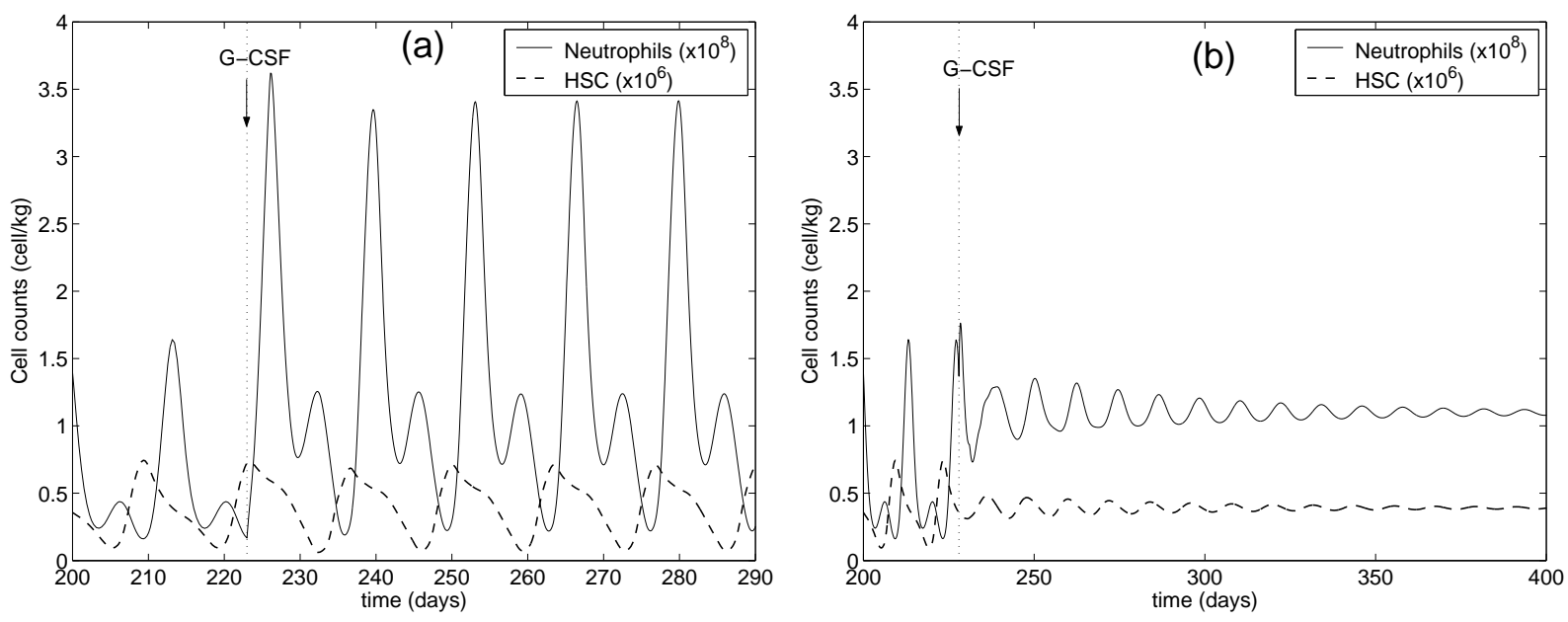

Figure 6: Daily G-CSF treatment when two possible behaviours can be seen depending on the starting day of treatment: (a) Large amplitude oscillations (treatment started at the ANC nadir (day 0)). (b) Stabilization of the ANC above the neutropenia level (starting day was 5 days after the ANC nadir). From [62].

increases toward 1, the steady state becomes stable at the Hopf bifurcation point (point $O$ ) and the amplitude of the limit cycle becomes larger and larger while its period decreases (not seen from the figure). This agrees with clinical data since G-CSF administration is generally associated with an increased amplitude and a decreased period of the oscillations $[63,64]$. However, some cases have been reported in the literature in which G-CSF treatment abolished significant oscillations $[9,63,64]$. Interestingly, the model accounts for this effect of G-CSF administration. Indeed, at $T=1$, one can see that a stable steady state (corresponding to annihilation of oscillations) coexists with a stable large amplitude oscillations (corresponding to large oscillations). This bistability in the system is very interesting since it suggests that by properly designing the treatment administration scheme, one might stabilize the neutrophil count to a desirable level and could potentially reduce the amount of G-CSF required in treatment. In [62], the authors exploited this bistability and showed that, depending on the starting date of the G-CSF treatment, the neutrophil count could either stabilize or show large amplitude oscillations (see Figure 6). Using computer simulations, they also showed that other G-CSF treatment schemes (such as administering G-CSF every other day) could be effective while using less G-CSF, hence reducing the cost of treatment and side effects for patients.

The [56] model grasped the essential features of the system while being simple enough to carry out the detailed analysis and simulations presented in [62]. It gave insight into the dynamics of the system but it had two major shortcomings. First, the model included neither erythrocyte nor platelet dynamics even though clinical data indicates oscillations in those cell lines in CN patients. Thus it is not known if the results would be consistent with observed platelet and reticulocyte data. Second, the simulations did not take into account 
the pharmacokinetics of G-CSF.

\subsubsection{A more sophisticated model}

G-CSF is typically injected and moves into the circulation (where it is degraded) and also has a saturable clearance mediated by circulating neutrophils. Due to the importance of G-CSF in treating bone marrow transplant and chemotherapy patients, there have been a variety of mathematical models of G-CSF kinetics [65] - [69]. In the hematological models described above, G-CSF is included implicitly using constant parameters such as neutrophil apoptosis and the maximal rate of differentiation into the neutrophil line [56, 60].

In [70] a pharmacokinetic model of G-CSF is coupled to the model presented in [60] by tying circulating G-CSF serum levels to the parameters most important in modeling G-CSF. These parameters $A_{N}, \gamma_{S}, \theta_{1}$, which were constant in earlier models, now become time-dependent and are functions the serum G-CSF concentration. The authors begin with model simulations that match neutrophil data for cyclic neutropenic gray collies, and explore the effects of applying different treatment schedules in the new model. The inclusion of GCSF kinetics affects the structure of the model, adding two more differential equations to Eq. 3.6. Despite this change, the fits to observed data, which resulted from automated fitting in [60], remain very good. Furthermore, the new model with G-CSF kinetics included preserves the bistability of periodic solutions, along with a locally stable steady solution, of the models in Eq. 3.2 and 3.3, i.e. the model of [56], and 3.6 from [60]. The preservation of the quantitative fits to data, and the qualitative features of the dynamics, is encouraging. Indeed, these are properties that should be preserved by refinements to the model.

Because the model with G-CSF kinetics included has several locally stable solutions, it has the capacity for the same kind of "branch switching" behavior that Eq. 3.6 has. In other words, different initial conditions or temporary interventions may lead to dramatically different long-term behaviors. In particular, in [70], the authors explore changing the period of G-CSF treatment (daily, every other day, and every third day). They also explore changing only the time at which treatment is first initiated. They found that both can significantly change the nature of the oscillations. In particular, there was one dog for whom varying only the time within the neutropenic cycle that treatment was initiated significantly reduced the amplitude of oscillations. Figure 7 shows the consequences of different treatment schemes with the model of [70].

In summary, there are indications from several mathematical models that varying the time of onset of G-CSF treatment, and the schedule of G-CSF delivery, can have surprisingly significant and long-term effects on the neutrophil oscillations.

\section{Discussion and conclusions}

Cyclic neutropenia is a perfect example of a dynamical disease-one in which there is a bifurcation (qualitative change) in the dynamics of an underlying physiological process in response to a change in a physiological parameter, c.f. [71] - [73]. In the case of cyclic neutropenia the 

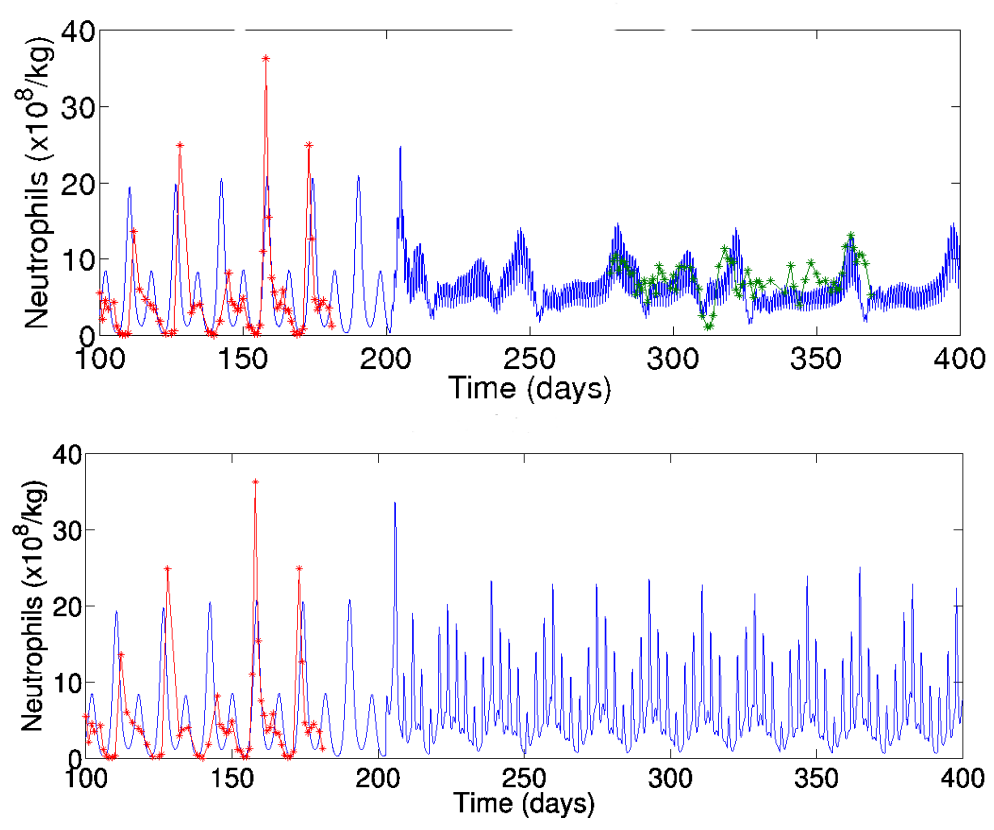

Figure 7: Consequences of different treatment schedules in the model of [70], showing data for the untreated and treated cases in the top plot. The top plot shows daily G-CSF injections, and the lower plots shows simulated oscillations assuming that G-CSF was delivered every 3 days instead of each day.

bifurcation is apparently due to a change in the rate of apoptosis in neutrophil precursors that triggers the interesting dynamics seen clinically in humans, and in the laboratory in grey collies.

Here we have reviewed an extensive and rich literature of experimental and clinical observations of this fascinating disease, as well as the attempts by biomathematicians to model and understand the observed dynamics. We hope that we have managed to highlight the torturous and convoluted path of these modeling efforts that eventually led to the correct prediction of the underlying pathophysiological mechanisms operative in cyclic neutropenia.

\section{Acknowledgements.}

This work was supported by the Natural Sciences and Engineering Research Council (NSERC, Canada) and the Mathematics of Information Technology and Complex Systems (MITACS, Canada). 


\section{References}

[1] D.C. Dale, W.P. Hammond. Cyclic neutropenia: A clinical review. Blood Rev., 2 (1998), 178-185.

[2] D.C. Dale, A.A. Bolyard, A. Aprikyan. Cyclic neutropenia. Semin. Hematol., 39 (2002), 89-94.

[3] D.G. Wright, D.C. Dale, A.S. Fauci, S.M. Wolff. Human cyclic neutropenia: clinical review and long term follow up of patients. Medicine, 60 (1981), 1-13.

[4] S.E. Palmer, K. Stephens, D.C. Dale. Genetics, phenotype, and natural history of autosomal dominant cyclic hematopoiesis. Am J Med Genet., 66 (1996), 413-422.

[5] C. Scully. Clinical practice. aphthous ulceration. N Engl J Med., 355 (2006), 165-172.

[6] G. Bar-Joseph, M. Halberthal, Y. Sweed, V. Bialik, O. Shoshani, A. Etzioni. Clostridium septicum infection in children with cyclic neutropenia. Pediatrics, 131 (1997), 317-319.

[7] C.L. Smith-Slatas, M. Bourque, J.C. Salazar. Clostridium septicum infections in children: a case report and review of the literature. Pediatrics, 117 (2006), 796-805.

[8] D.C. Dale, M.A. Bonilla, M.W. Davis, A. Nakanishi, W.P. Hammond, J. Kurtzberg, W. Wang, A. Jakubowski, E. Winton, e. a. P. Lalezari. A randomized controlled phase III trial of recombinant human granulocyte colony-stimulating factor (filgrastim) for treatment of severe chronic neutropenia. Blood, 81 (1993), 2496-2502.

[9] W.P. Hammond, T.H. Price, L.M. Souza, D.C. Dale. Treatment of cyclic neutropenia with granulocyte colony stimulating factor. New Eng. J. Med., 320 (1989), 1306-1311.

[10] K. Kaushansky. Lineage-specific hematopoietic growth factors. New Engl J Med., 354 (2006), 2034-2045.

[11] K. Hubel, D.C. Dale, W.C. Liles. Thereapeutic use of cytokines to modulate phagocyte function for the treatment of infectious diseases: current status of granulocyte colonystimulating factor, macrophage colony-stimulating factor, and interferon-gamma. J. Infect. Dis., 185 (2002), 1490-1501.

[12] D.C. Dale, T.E. Cottle, C.J. Fier, A.A. Bolyard, M.A. Bonilla, L.A. Boxer, B. Cham, M.H. Freedman, G. Kannourakis, S.E. Kinsey, R. Davis, D. Scarlata, B. Schwinzer, C. Zeidler, K. Welte. Severe chronic neutropenia: treatment and follow-up of patients in the Severe Chronic Neutropenia International Registry. Am J Hematol., 72 (2003), 82-93.

[13] A.A. Morley, J.P. Carew, A.G. Baikie. Familial cyclical neutropenia. Br J Haematol., 13 (1967), 719-738. 
[14] M. Horwitz, K. Benson, R. Person, A. Aprikyan, D. Dale. Mutations in ELA2, encoding neutrophil elastase, define a 21-day biological clock in cyclic haematopoiesis. Nat. Genet., 23 (1999), 433-436.

[15] H.A. Reimann, C.T. de Beradinis. Periodic (cyclic) neutropenia, an entity. A collection of sixteen cases. Blood, 4 (1949), 1109-1116.

[16] P.S. Rosenberg, B.P. Alter, A.A. Bolyard, M.A. Bonilla, L.A. Boxer, B. Cham, C. Fier, M. Freedman, G. Kannourakis, S. Kinsey, B. Schwinzer, C. Zeidler, K. Welte, D.C. Dale. Severe chronic neutropenia international registry, the incidence of leukemia and mortality from sepsis in patients with severe congenital neutropenia receiving long-term G-CSF therapy. Blood, 107 (2006), 4628-4635.

[17] D. Guerry, D.C. Dale, M. Omine, S. Perry, S.M. Wolff. Periodic hematopoiesis in human cyclic neutropenia. J. Clin. Inves., 52 (1973), 3220-3230.

[18] A.A. Aprikyan, W.C. Liles, E. Rodger, M. Jonas, E. Chi, D. C. Dale. Impaired survival of bone marrow hematopoietic progenitor cells in cyclic neutropenia. Blood, 97 (2001), 147-153.

[19] D. Garwicz, A. Lennartsson, S. Jacobsen, U. Gullberg, A. Lindmark. Biosynthetic profiles of neutrophil serine proteases in a human bone marrow-derived cellular myeloid differentiation model. Haematologica, 90 (2005), 38-44.

[20] M.G. Hunter, L.J. Druhan, P.R. Massullo, B.R. Avalos. Proteolytic cleavage of granulocyte colony-stimulating factor and its receptor by neutrophil elastase induces growth inhibition and decreased cell surface expression of the granulocyte colony-stimulating factor receptor. Am J. Hematol., 74 (2003), 149-55.

[21] I. Kollner, B. Sodeik, S. Schreek, H. Heyn, N. von Neuhoff, M. Germeshausen, C. Zeidler, M. Kruger, B. Schlegelberger, K. Welte, C. Beger. Mutations in neutrophil elastase causing congenital neutropenia lead to cytoplasmic protein accumulation and induction of the unfolded protein response. Blood, 108 (2006), 493-500.

[22] D.C. Dale, R.E. Person, A.A. Bolyard, A. Aprikyan, C. Bos, M.A. Bonilla, L.A. Boxer, G. Kannourakis, C. Zeidler, K. Welte, K.F. Benson, M. Horwitz. Mutations in the gene encoding neutrophil elastase in congenital and cyclic neutropenia. Blood, 96 (2000), $2317-2322$.

[23] M.A. Murakami, D.S. Grenda, J. Ghatak, L.A. Boxer, D.C. Dale, M.C. Dinauer, A.A. Bolyard, D.C. Link. Mutations of the ELA22 gene found in patients with severe congenital neutropenia induce the unfolded protein response and cellular apoptosis. ASH Annual Meeting Abstracts, 108 (2006), 151. 
[24] C. Haurie, D.C. Dale, M.C. Mackey. Occurrence of periodic oscillations in the differential blood counts of congenital, idiopathic, and cyclical neutropenia patients before and during treatment with G-CSF. Exp Hematol., 27 (1999), 401-409.

[25] D.C. Dale, A. Aprikyan. Cyclic and congenital neutropenia due to defects in neutrophil elastase. In: Primary Immunodeficiency Diseases (P.J. Ochs, H.D., Smith C.I.D., ed.), 2006.

[26] D.C. Dale, S.B. Ward, H.R. Kimball, S.M. Wolff. Studies of neutrophil production and turnover in grey collie dogs with cyclic neutropenia. Journal of Clinical Investigation, 51 (1972), 2190-2196.

[27] D.C. Dale, D.W. Alling, S.M. Wolff. Cyclic hematopoiesis: the mechanism of cyclic neutropenia in grey collie dogs. Journal of Clinical Investigation, 51 (1972), 2197-2204.

[28] R.E. Scott, D.C. Dale, A.S. Rosenthal, S.M. Wolff. Cyclic neutropenia in grey collie dogs. Ultrastructural evidence for abnormal neutrophil granulopoiesis. Lab Invest., 28 (1973), 514-525.

[29] K.F. Benson, F.Q. Li, R.E. Person, D. Albani, Z. Duan, J. Wechsler, K. Meade-White, K. Williams, G.M. Acland, G. Niemeyer, C.D. Lothrop, M. Horwitz. Mutations associated with neutropenia in dogs and humans disrupt intracellular transport of neutrophil elastase. Nat. Genet., 90 (2003), 90-96.

[30] W.P. Hammond, T.C. Boone, R.E. Donahue, L.M. Souza, D.C. Dale. Comparison of treatment of canine cyclic hematopoiesis with recombinant human granulocytemacrophage colony-stimulating factor (GM-CSF), G-CSF, Interleukin-3, and Canine G-CSF. Blood, 76 (1990), 523-532.

[31] O. Yanay, M. Brzezinski, J. Christensen, D. Liggitt, D.C. Dale, W.R. Osborne. An adult dog with cyclic neutropenia treated by lentivirus-mediated delivery of granulocyte colony-stimulating factor. Hum. Gene Ther., 17 (2006), 464-469.

[32] C.D.R. Dunn. Cyclic hematopoiesis: the biomathematics. Exp. Hematol., 11 (1983), 779-791.

[33] G. Fisher. An introduction to chaos theory and some haematological applications. Comp. Haematol. Int, 3 (1993), 43-51.

[34] C. Haurie, M.C. Mackey, D.C. Dale. Cyclical neutropenia and other periodic hematological diseases: a review of mechanisms and mathematical models. Blood, 92 (1998), 2629-2640.

[35] M.C. Mackey. Mathematical models of hematopoietic cell replication control. In: The Art of Mathematical Modeling: Case Studies in Ecology, Physiology and Biofluids (Eds. H. Othmer, F. Adler, M. Lewis, J. Dallon), New York: Prentice Hall, 1996. 149-178. 
[36] N.D. Kazarinoff, P. van den Driessche. Control of oscillations in hematopoiesis. Science, 203 (1979), 1348-1350.

[37] E.A. King-Smith, A. Morley. Computer simulation of granulopoiesis: Normal and impaired granulopoiesis. Blood, 36 (1970), 254-262.

[38] N. MacDonald. Cyclical neutropenia: models with two cell types and two time lags. In: Biomathematics and Cell Kinetics (Eds. A. Valleron, P. Macdonald ), Elsevier/NorthHolland, Amsterdam, 1978, 287-295.

[39] A. Morley. Cyclic hemopoiesis and feedback control. Blood Cells, 5 (1979), 283-296.

[40] A. Morley, E.A. King-Smith, F. Stohlman. The oscillatory nature of hemopoiesis. In: Hemopoietic Cellular Proliferation (Ed. F. Stohlman ), Grune \& Stratton, New York, $1969,3-14$.

[41] A. Morley, F. Stohlman. Cyclophosphamide induced cyclical neutropenia. New England Journal of Medicine, 282 (1970), 643-646.

[42] J. Reeve. An analogue model of granulopoiesis for the analysis of isotopic and other data obtained in the non-steady state. Br. J. Haematol., 25 (1973), 15-32.

[43] S. Schmitz. Ein mathematisches Modell der zyklischen Haemopoese. PhD thesis, Universitat Koln, 1988.

[44] S. Schmitz, H. Franke, J. Brusis, H.E.Wichmann. Quantification of the cell kinetic effects of G-CSF using a model of human granulopoiesis. Exp. Hematol., 21 (1993), 755-760.

[45] S. Schmitz, H. Franke, M. Loeffler, H.E. Wichmann, V. Diehl. Reduced variance of bone-marrow transit time of granulopoiesis: a possible pathomechanism of human cyclic neutropenia. Cell Prolif., 27 (1994), 655-667.

[46] S. Schmitz, H. Franke, H.E. Wichmann, V. Diehl. The effect of continuous G-CSF application in human cyclic neutropenia: a model analysis. Br. J. Haematol., 90 (1995), 41-47.

[47] S. Schmitz, M. Loeffler, J.B. Jones, R.D. Lange, H.E. Wichmann. Synchrony of bone marrow proliferation and maturation as the origin of cyclic haemopoiesis. Cell Tissue Kinet., 23 (1990), 425-441.

[48] D. Shvitra, R. Laugalys, Y.S. Kolesov. Mathematical modeling of the production of white blood cells. In: Mathematical Modeling in Immunology and Medicine (Eds. G. Marchuk, L. Belykh), North-Holland, Amsterdam, 1983, 211-223.

[49] G.K. von Schulthess, N.A. Mazer. Cyclic neutropenia (CN): a clue to the control of granulopoiesis. Blood, 59 (1982), 27-37. 
[50] H.E. Wichmann, M. Loeffler, S. Schmitz. A concept of hemopoietic regulation and its biomathematical realization. Blood Cells, 14 (1988), 411-429.

[51] M.C. Mackey. A unified hypothesis for the origin of aplastic anemia and periodic haematopoiesis. Blood, 51 (1978), 941-956.

[52] M.C. Mackey. Dynamic haematological disorders of stem cell origin. In: Biophysical and Biochemical Information Transfer in Recognition (Eds. J.G. Vassileva-Popova, E.V. Jensen), Plenum Publishing Corp., New York, 1979, 373-409.

[53] J.G. Milton, M.C. Mackey. Periodic haematological diseases: mystical entities or dynamical disorders?. J. Roy. Coll. Phys. (Lond.), 23 (1989), 236-241.

[54] T. Hearn, C. Haurie, M. Mackey. Cyclical neutropenia and the peripherial control of white blood cell production. J. Theor. Biol., 192 (1998), 167-181.

[55] C. Haurie, D.C. Dale, R. Rudnicki, M.C. Mackey. Modeling complex neutrophil dynamics in the grey collie. J. Theor. Biol., 204 (2000), 505-519.

[56] S. Bernard, J. Belair, M. Mackey. Oscillations in cyclical neutropenia: new evidence based on mathematical modeling. J. Theor. Bio., 223 (2003).

[57] C. Colijn, M. Mackey. A mathematical model of hematopoiesis: I. Periodic chronic myelogenous leukemia. J. Theor. Biol., 237 (2005), No. 2.

[58] J.M. Mahaffy, J. Belair, M.C. Mackey. Hematopoietic model with moving boundary condition and state dependent delay. J. Theor. Biol., 190 (1998), 135-146.

[59] M. Santillan, J. Belair, J. Mahaffy, M. Mackey. Regulation of platelet production: the normal response to perturbation and cyclical platelet disease. J. Theor. Biol., 206 (2000), 585-603.

[60] C. Colijn, M. Mackey. A mathematical model of hematopoiesis: II. Cyclical neutropenia. J. Theor. Biol., 237 (2005), No. 2.

[61] C. Colijn, M. Mackey. Bifurcation and bistability in a model of hematopoietic regulation. SIAM J. Appl. Dynam. Sys., 6 (2007), 378-394.

[62] C. Foley, S. Bernard, M. Mackey. Cost-effective G-CSF therapy strategies for cyclical neutropenia: mathematical modelling based hypotheses. J. Theor. Biol., 238 (2006), 754763.

[63] C. Haurie, M.C. Mackey, D.C. Dale. Occurrence of periodic oscillations in the differential blood counts of congenital, idiopathic, and cyclical neutropenic patients before and during treatment with G-CSF. Exper. Hematol., 27 (1999), 401-409. 
[64] C. Haurie, R. Person, M.C. Mackey, D.C. Dale. Hematopoietic dynamics in grey collies. Exper. Hematol., 27 (1999), 1139-1148.

[65] N. Hayashi, H. Aso, M. Higashida, H. Kinoshita, S. Ohdo, E. Yukawa, S. Higuchi. Estimation of RHG-CSF absorption kinetics after subcutaneous administration using a modified Wagner-Nelson method with a nonlinear elimination model. Eur. J. Pharm. Sci., 15 (2001), 151-158.

[66] T. Kuwabara, Y. Kato, S. Kobayashi, H. Suzuki, Y. Sugiyama. Nonlinear pharmacokinetics of a recombinant human granulocyte colony-stimulating factor derivative (nartograstim): species differences among rats, monkeys and humans. J. Pharmacol. Exp. Ther., 271 (1994), No. 3, 1535-1543.

[67] N. Stute, V. Santana, J. Rodman, M. Schell, J. Ihle, W. Evans. Pharmacokinetics of subcutaneous recombinant human granulocyte colony-stimulating factor in children. Blood, 79 (1992), No. 11, 2849-2854.

[68] H. Takatani, H. Soda, M. Fukuda, M. Watanabe, A. Kinoshita, T. Nakamura, M. Oka. Levels of recombinant human granulocyte colony stimulating factor in serum are inversely correlated with circulating neutrophil counts. Antimicrob. Agents Chemother., 40 (1996), 988-991.

[69] V. Vainstein, Y. Ginosara, M. Shohamb, D. Ranmara, A. Ianovskib, Z. Agur. The complex effect of granulocyte colony-stimulating factor on human granulopoiesis analyzed by a new physiologically-based mathematical model. J. Theor. Biol., 234 (2005), No. 3, 311-327.

[70] C. Colijn, C. Foley, M. Mackey. G-CSF treatment of cyclical neutropenia: an inclusive mathematical model. J. Exp. Hematol., 35 (2007), 898-907.

[71] M. C. Mackey, L. Glass. Oscillations and chaos in physiological control systems. Science, 197 (1977), 287-289.

[72] L. Glass, M. C. Mackey. Pathological conditions resulting from instabilities in control systems. Ann. N.Y. Acad. Sci., 316 (1979), 214-235.

[73] L. Glass, M.C. Mackey. From clocks to chaos: The rhythms of life. Princeton University Press, Princeton, 1988. 